\title{
Interaction between localized and delocalized surface plasmon polariton modes in a metallic photonic crystal
}

\author{
A. Christ ${ }^{*}, 1,2$, T. Zentgraf ${ }^{1}$, S. G. Tikhodeev ${ }^{3}$, N. A. Gippius ${ }^{3}$, O. J. F. Martin ${ }^{2}$, J. Kuhl ${ }^{1}$, \\ and H. Giessen ${ }^{4}$ \\ ${ }^{1}$ Max-Planck-Institut für Festkörperforschung, 70569 Stuttgart, Germany \\ ${ }^{2}$ Nanophotonics and Metrology Laboratory, Swiss Federal Institute of Technology (EPFL), \\ 1015 Lausanne, Switzerland \\ 3 A. M. Prokhorov General Physics Institute RAS, Moscow 119991, Russia \\ ${ }^{4} 4^{\text {th }}$ Physics Institute, University of Stuttgart, 70550 Stuttgart, Germany
}

Received 20 February 2006, revised 18 April 2006, accepted 18 April 2006

Published online 12 July 2006

PACS 42.70.Qs, 73.20.Mf, 78.66.--w

We experimentally and theoretically study the controlled coupling between localized and delocalized surface plasmon modes supported by a multilayer metallic photonic crystal slab. The model system to visualize the interaction phenomena consists of a gold nanowire grating and a spatially separated homogeneous silver film. We show that plasmon-plasmon coupling leads to drastic modification of the optical properties in dependence on the chosen geometrical parameters. Strong coupling and plasmon hybridization can be clearly observed. The numerical calculations reveal excellent agreement with the experiments.

It is well-known that the interaction between electromagnetic radiation and electric dipole resonances can be strongly altered in the presence of a nearby reflecting metal surface [1]. Shifts of the resonance frequencies as well as lifetime modifications have been observed in dependence on the dipole-surface separation. In particular, systems which are composed of a dipole-active layer (e.g., metal island films) placed in front of a metal mirror have been extensively investigated [2,3]. A very interesting alternative situation may arise when the studies are extended to more complex structures which incorporate a spatial ordering of the dipole ensemble, e.g., a periodic dipole arrangement [4]. We propose a metallic model system to study the influence of such periodicity on the optical response of the multilayer system. Noble metal nanowires are used as elementary dipole oscillators in our experimental and theoretical studies. The optical response of the individual metal nanowires in TM polarization (magnetic field oriented parallel to the wires) is generally characterized by localized particle plasmon resonances which are a consequence of the collective oscillation of their conduction band electrons.

A schematic view of the metallic photonic crystal slab is depicted in Fig. 1(a). Electron-beam lithography and thermal evaporation are used to deposit a one-dimensional gold nanowire grating on top of the $\mathrm{SiO}_{2}$ spacer layer. The thin spacer layer of thickness $L_{s p}$ spatially separates the gold grating from the underlying 20-nm-thick homogeneous silver film covering the quartz substrate. The grating extension was restricted to $100 \times 100 \mu \mathrm{m}^{2}$. A scattering-matrix formalism has been employed for all numerical calculations [5]. Only the geometrical properties of the structure and the dielectric susceptibilities of the constituent materials are required as input parameters [6]. It is important to remark that an optimized gold nanowire cross-section of $100 \times 15 \mathrm{~nm}^{2}$ has been used for all scattering-matrix based calculations.

" Corresponding author: e-mail: andre.christ@epfl.ch, Phone: +41 21693 3968, Fax: +41 216932614 
(a)

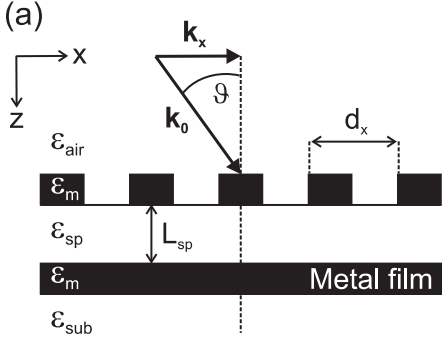

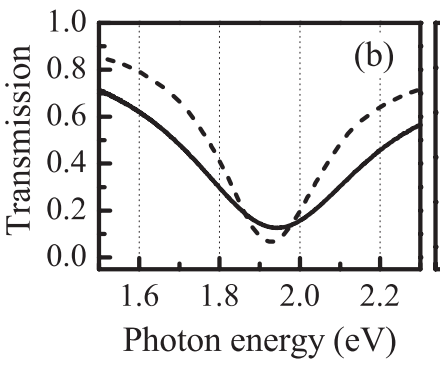

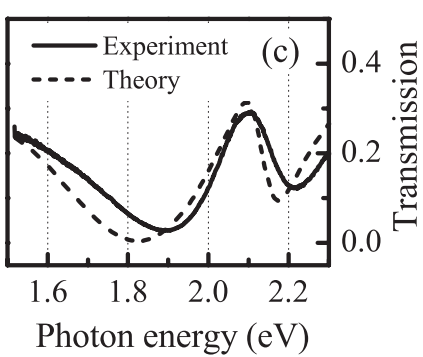

Fig. 1 (a) Schematic view of the metallic photonic crystal slab structure. The gold nanowire array and the nearby 20 -nm-thick silver film are separated by a dielectric spacer layer of thickness $L_{s p}$. Experimentally (solid lines) and theoretically (dashed lines) obtained transmission spectra are displayed in panels (b) and (c). Spectra of a pure gold grating (b) and of a metallic multilayer structure (c) with $L_{s p}=70 \mathrm{~nm}$ are compared. The spectra $\left(d_{x}=300 \mathrm{~nm}\right)$ are shown for TM polarization and normal light incidence.

This slight deviation from the experimentally determined nanowire cross-section of $100 \times 20 \mathrm{~nm}^{2}$ might be caused by uncertainties in the exact determination of the gold susceptibility.

The optical response of a simple gold grating without the nearby silver film is shown in panel (b) of Fig. 1 for TM polarization and normal light incidence. The localized plasmon resonance of the nanowires is clearly observable as a single transmission minimum. Note that the measured plasmon linewidth is slightly increased due to the influence of inhomogeneous broadening. The silver film induced modification of the spectra is highlighted in panel (c) of Fig. 1. Instead of the single localized nanowire plasmon resonance, two transmission minima can be clearly observed in case of a stacked multilayer design. The experimentally and theoretically observed double peak structure is hence a clear signature of the induced dipole-film interaction.

We will start with a purely theoretical discussion based on numerical simulations to clarify the physical nature of the observed resonances. Actually, it is very instructive to analyze the optical response of a slightly different grating geometry first. We therefore recapitulate the fundamental properties of a stacked double grating structure based on laterally coupled wire pairs. Figure 2 (a) simultaneously dis-

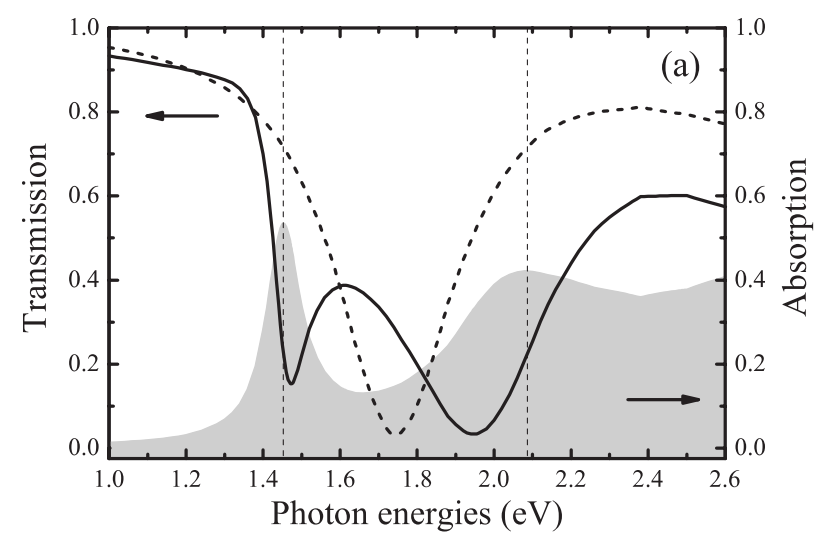

(b)

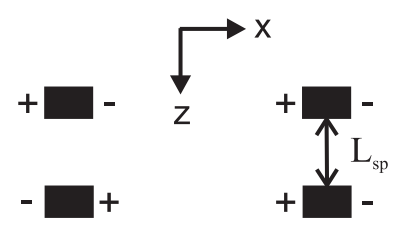

Antisymmetric

Symmetric

plasmon mode

plasmon mode

Fig. 2 (a) Calculated spectra of two different grating geometries $\left(d_{x}=300 \mathrm{~nm}\right)$ for TM polarization and normal light incidence. The transmission spectrum of a pure gold grating (dashed line) is compared with the spectrum of a similar double grating structure (solid line) with $L_{s p}=40 \mathrm{~nm}$. Additionally, the calculated absorption spectrum (shaded area) of the coupled grating structure is plotted. The characteristic charge distribution of the fundamental plasmon modes in the coupled nanowire geometry (wire-wire coupling) is pictorially displayed in panel (b). 

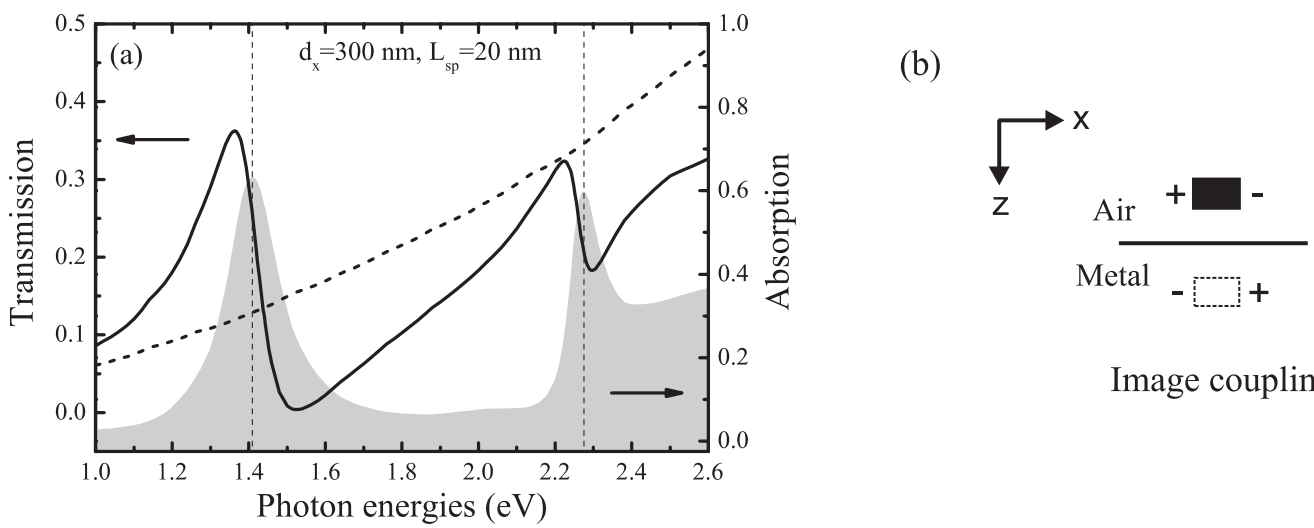

Image coupling

Fig. 3 (a) Calculated transmission (solid line) and absorption spectra (shaded area) of a multilayer photonic crystal slab structure for TM polarization and normal light incidence. Spectra for a structure with a spacer layer thickness of $20 \mathrm{~nm}$ are shown $\left(d_{x}=300 \mathrm{~nm}\right)$. The transmission spectrum of a pure 20-nmthick silver film without nearby grating is plotted (dashed line) as a reference. The characteristic charge distribution of the fundamental plasmon mode in the grating-film geometry is pictorially displayed in panel (b).

plays the transmission spectra of a simple planar gold grating and of a more complex double layer structure (i.e., two stacked gold nanowire arrays covered by quartz and spatially separated by a 40-nm-thick spacer layer). As schematically illustrated in panel (b) of Fig. 2, the observed splitting into two transmission minima in case of the stacked grating structure (solid line) is a clear result of plasmon hybridization. Characterized by their specific charge distributions, antisymmetric and symmetric plasmon modes are supported simultaneously [7]. While the antisymmetric or magnetic plasmon mode is redshifted in comparison to the bare resonance (dashed line) of the single layer grating, the spectral position of the symmetric or electric plasmon resonance is increased in energy. Generally, the observed spectral splitting indicates a strong spacer layer dependence (not shown).

The optical response of the multilayer grating-film structure $\left(L_{s p}=20 \mathrm{~nm}, d_{x}=300 \mathrm{~nm}\right)$ on the other hand is exemplary displayed in Fig. 3 (a). Although the spectra look quite different, some of the characteristic properties of the double layer grating, i.e., wire pair geometry are reflected. In particular, the magnetic plasmon mode is directly mimicked by the interaction between the wire plasmon and its own image as sketched in Fig. 3 (b). The symmetric mode of Fig. 2 (b) does not exist in case of image coupling. The bare localized magnetic mode of the grating-film structure can be found at $1.41 \mathrm{eV}$ in Fig. 3 (a). Note that the specific nature of the localized magnetic plasmon mode (i.e., inductor-capacitor circuit) can be directly visualized by plotting the numerically calculated field distribution [8]. However, a second pronounced mode can be identified at $2.28 \mathrm{eV}$. This resonance is caused by the grating-induced excitation of an extended surface plasmon mode at the nearby silver film. The electromagnetic coupling between the supported localized and delocalized plasmon modes can be controlled by changing the spacer layer thickness, the grating period, or the illumination angle. Here, we restrict all further discussion to the influence of the spacer layer thickness. We have to remark that the fundamental phenomenon of plasmon-plasmon interaction is directly related to the so-called waveguide-plasmon polariton formation in metallo-dielectric photonic crystal slabs [9].

The influence of the spacer layer on the optical properties is highlighted in panels (a) and (b) of Fig. 4. Calculated transmission and absorption spectra are shown for a grating-film structure with a constant nanowire period of $d_{x}=300 \mathrm{~nm}$. Both mirror induced shifts of the localized mode as well as its interaction with the Bragg surface mode can be clearly observed. The lower transmission minimum can be associated with the approximate position of the localized plasmon mode. Its bare position (i.e., neglecting the silver film) is indicated by the vertical dotted line. The mode is strongly redshifted when decreasing the spacer layer thickness. This phenomenon can be explained by the enhanced near-field interaction 

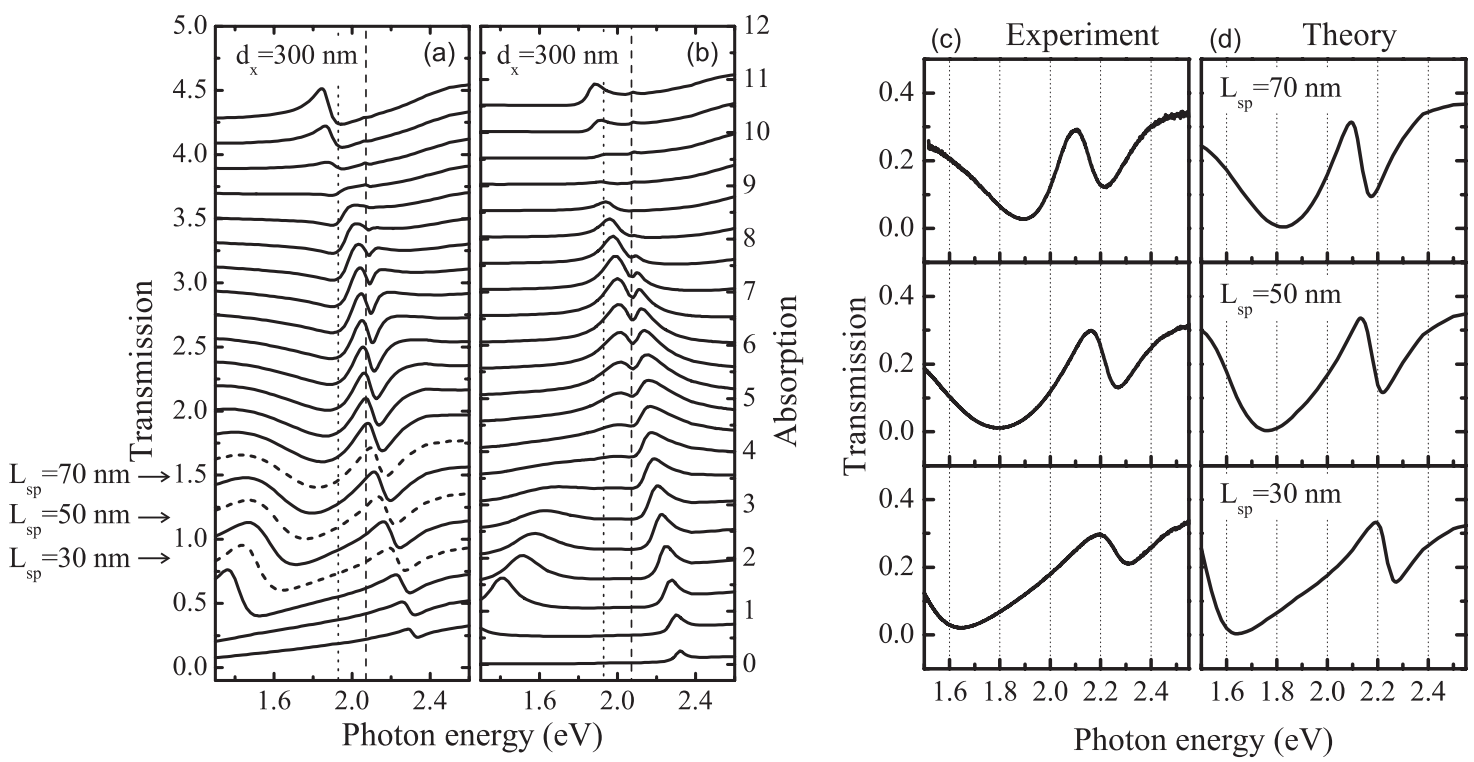

Fig. 4 Calculated transmission (a) and absorption (b) spectra of a grating-film structure $\left(d_{x}=300 \mathrm{~nm}\right)$ in dependence on the spacer layer thickness. The spectra are shown for normal light incidence and TM polarization. From bottom to top, the spacer layer thickness is increased from $0 \mathrm{~nm}$ to $210 \mathrm{~nm}$ in steps of $10 \mathrm{~nm}$. The spectral position of the supported bare modes are indicated by vertical lines (dotted line: localized nanowire plasmon mode, dashed line: Bragg-induced surface plasmon resonance). The individual spectra are shifted upwards for clarity. In panel (c) and (d), the experimental and theoretical transmission spectra of structures with a spacer layer thickness of $30 \mathrm{~nm}$, $50 \mathrm{~nm}$, and $70 \mathrm{~nm}$ are compared $\left(d_{x}=300 \mathrm{~nm}\right)$, respectively.

between the localized nanowire plasmon and its own induced image. The linear restoring forces which arise due to the collectively displaced nanowire conduction band electrons are hence effectively reduced. On the other hand, the upper weaker pronounced transmission minimum is blueshifted simultaneously. This shift is related to the gradually modified character of the involved grating-induced surface plasmon mode. The Bragg surface plasmon mode which is mainly localized at the silver-substrate interface (corresponding to a negligible thickness of the spacer layer) is transferred into the short range surface plasmon mode of the more symmetric film structure (corresponding to a thin silver film between quartz half spaces, indicated by the dashed vertical line). Note that the coupling between localized and delocalized modes leads to an enhanced resonant absorption for structures with intermediate spacer layer thicknesses $\left(L_{s p} \approx 100 \mathrm{~nm}\right)$. In panels (c) and (d) of Fig. 4, three characteristic transmission spectra of the displayed series (dashed lines in panel (a)) are finally compared with experimental results to verify the numerical simulations. The calculations reveal excellent agreement with the shown experimentally measured spectra.

In conclusion, we have analyzed the electromagnetic interaction between a localized particle plasmon resonance and a delocalized surface plasmon mode in a multilayer photonic crystal slab. The experimental and theoretical results clearly reveal a characteristic mixing of the plasmon modes. The presented interaction phenomena are important for the optimization of future plasmonic nanodevices.

Acknowledgements This work was financially supported by the German Federal Ministry of Education and Research (FKZ 13N8340/1), the Deutsche Forschungsgemeinschaft (Priority Program SPP1113, FOR557), the Russian Academy of Science (Program "Low-dimensional quantum structures", 2.3), the Russian Foundation for Basic Research (03-02-17314), and the European Commission (FP6-2002-IST-1-507879). The authors acknowledge the support of K. von Klitzing. 


\section{References}

[1] W. L. Barnes, J. Mod. Opt. 445, 661 (1998).

[2] W. R. Holland and D. G. Hall, Phys. Rev. B 27, 7765 (1983).

[3] W. R. Holland and D. G. Hall, Phys. Rev. Lett. 52, 1041 (1984).

[4] J. Cesario, R. Quidant, G. Badenes, and S. Enoch, Opt. Lett. 30, 3404 (2005).

[5] S. G. Tikhodeev et al., Phys. Rev. B 66, 045102 (2002).

[6] P. B. Johnson and R. W. Christy, Phys. Rev. B 6, 4370 (1972).

[7] G. Dolling et al., Opt. Lett. 30, 3198 (2005).

[8] A. Christ et al., submitted (2006).

[9] A. Christ et al., Phys. Rev. Lett. 91, 183901 (2003). 